\title{
Durabilidad y acústica del concreto con escoria de cubilote como reemplazo del agregado fino
}

\section{Durability and acoustics of concrete with slag of cupola furnace as}

fine aggregate replacement

\section{Ricardo Alfredo Cruz Hernández}

Ingeniero civil, doctor en Ciencias Técnicas, profesor de la Universidad Industrial de Santander, Bucaramanga, Director del Grupo de Investigación en Estructuras y Materiales de Construcción, Colombia. Contacto: racruz@uis.edu.co

\section{Carlos Mauricio Pico Cortés}

Ingeniero civil, especialista en Análisis y Diseño de Estructuras, investigador de la Universidad Industrial de Santander, Bucaramanga, Colombia.

Contacto: carlos.pico1@correo.uis.edu.co

\section{Ludwing Pérez Bustos}

Ingeniero civil, magíster en Ingeniería Civil, investigador de la Universidad Industrial de Santander, docente de la Universidad Industrial de Santander, Bucaramanga, Colombia. Contacto: jupebu26@hotmail.com

Fecha de recepción: 18 de julio de 2013

Fecha de aceptación: 30 de abril de 2014
Clasificación del artículo: investigación

Financiamiento: Vicerrectoría de Investigación y Extensión, Universidad Industrial de Santander, Bucaramanga

DOI: http://dx.doi.org/10.14483/udistrital.jour.tecnura.2015.1.a02

Palabras clave: agregado fino de escoria, carbonatación, coeficiente de absorción acústica, reacción álcali-agregado, sulfatación.

Keywords: Alkali-aggregate reactions, carbonation, slag as fine aggregate, sound absorption coefficient, sulfation.

\section{RESUMEN}

En este trabajo se evaluó el comportamiento del concreto con escoria de horno de cubilote (EHC), triturada en porcentajes de sustitución de arena del $0,10,15$ y $20 \%$, sometido a ataques químicos acelerados de carbonatación, sulfatación y reac- ción álcali-agregado (RAA). Se determinaron las características de absorción del sonido del material a través del coeficiente de absorción acústica ( $\alpha$ ) y el índice de reducción de ruido (NRC). La carbonatación se evaluó por medio de una cámara cerrada con $70 \%$ de concentración de dióxido de carbono y condiciones de humedad relativa entre 
50 y $70 \%$. Los resultados mostraron que la profundidad de penetración de $\mathrm{CO}_{2}$ es menor mientras mayor es el porcentaje de sustitución. Para acelerar el ataque por sulfatos, se sumergieron probetas en solución acuosa de sulfato de sodio anhidro $\left(\mathrm{Na}_{2} \mathrm{SO}_{4}\right) 1 \mathrm{~N}$ con ciclos de humedecimiento y secado. Se determinó que el deterioro presentado en la pasta de concreto es directamente proporcional al porcentaje de sustitución. La aceleración de la RAA en el concreto se realizó sumergiendo probetas en una solución acuosa de hidróxido de sodio $(\mathrm{NaOH})$ durante dieciséis días. Se concluyó que la inclusión de EHC no es favorable por RAA. La medición del coeficiente de absorción acústica se realizó a través del método de tubo de impedancia, relacionando valores máximos y mínimos de amplitud de onda estacionaria. Los resultados mostraron que concretos de EHC con mayores sustituciones de arena por escoria son favorables para la absorción de ruidos en las edificaciones.

\section{ABSTRACT}

In this paper, it was evaluated the performance of concrete with crushed slag of cupola furnace $(\mathrm{SCF})$ as sand replacement in percentages of $0 \%$,
$10 \%, 15 \%$ and $20 \%$, subjected to accelerated chemical attacks of carbonation, sulfation and alkali-aggregate reaction (AAR). The sound absorption characteristics of the material were determined through the sound absorption coefficient $(\alpha)$, and the noise reduction coefficient (NRC). Carbonation was evaluated through a closed camera with the $70 \%$ concentration of carbon dioxide and conditions of relative humidity between $50 \%$ and $70 \%$. The results indicated that the penetration depth of $\mathrm{CO}_{2}$ is lower when greater the percentage of substitution is. To accelerate the attack by sulfates, specimens were immersed in aqueous solution of sodium sulfate anhydrous $\left(\mathrm{Na}_{2} \mathrm{SO}_{4}\right)$ $1 \mathrm{~N}$ with cycles of wetting and drying. It determined that the impairment presented in concrete paste is directly proportional to the percentage of sand replacement. The acceleration of the AAR in the concrete was carried out by immersing specimens in an aqueous solution of sodium hydroxide $(\mathrm{NaOH})$ for 16 days. The test concluded that the inclusion of SCF is not favorable for AAR. The measurement of sound absorption coefficient was taken by the method of impedance tube, relating minimum and maximum values of stationary wave amplitude. The results showed that SCF with higher sand replacement are favorable for the noise absorption in buildings.

\section{INTRODUCCIÓN}

La tecnología de materiales avanza constantemente en la búsqueda de estructuras cada vez más durables y confortables. Nuevas exigencias ambientales y tendencias comerciales vienen motivando, entre otras, a la industria del concreto a disminuir su impacto, reduciendo la emisión de $\mathrm{CO}_{2}$ y el consumo de materia prima natural (Anastasiou, Georgiadis y Stefanidou, 2014). La tendencia actual es el uso de materiales alternativos en la fabricación de concreto, por la ventaja económica que esto puede representar, además

del mejoramiento de algunas de sus propiedades. Se ha incrementado la durabilidad del concreto utilizando cenizas volantes (Blissett y Rowson, 2012) y escorias, sin detrimento de su resistencia. Incluso, se han obtenido mejores propiedades en comparación con concretos con cemento Portland (Mohammed y Pofale, 2012; Zakaria y Cabrera, 1996).

En los últimos años se han desarrollado investigaciones tendientes a ampliar el uso de los concretos con agregados alternativos, evaluando otras propiedades como las acústicas, térmicas y de re- 


\section{investigación}

sistencia al fuego. Se han evaluado las características acústicas de concretos, particularmente porosos (Kim y Lee, 2010; Doutres, Salissou, Atalla y Panneton, 2010; Park, Seo y Jun, 2005), celulares, espumosos (Ramamurthy, Kunhanandan e Indu Siva, 2009) y se han comparado con concretos normales. Además, se han realizado estudios relacionados con el aislamiento acústico de muros (Leiva, Solís-Guzman, Marrero y García Arena, 2013), placas de concreto (Pastor, García, Quintana y Peña, 2014) y pavimentos rígidos (Tiwari, Shukla y Bose, 2004). Doutres (2010) evaluó las propiedades acústicas de materiales porosos utilizando el método de tubo de impedancia con tres micrófonos y comparó los resultados obtenidos con otros métodos directos e inversos, entre ellos el método del tubo de impedancia que se usó en esta investigación.

Por otra parte, alrededor del mundo se han realizado aportes respecto a la utilización de la escoria como sustituto del cemento. La escoria ha sido utilizada como agregado grueso, con el fin de obtener concretos aligerados (Zaetang, Wongsa, Sata y Chindaprasirt, 2013), aunque puede llegar a ser un material más denso que otras alternativas (Naik, 2002). Baricová (2010) evaluó la resistencia a la compresión y encontró que un reemplazo de arena del $100 \%$ es inadecuado, pero un reemplazo del $20 \%$, además de tener una resistencia a la compresión similar a la de un concreto normal, favorece el aumento de la resistencia a la flexión.

Esta investigación contribuye al estudio de la durabilidad del concreto, por medio de la caracterización de la EHC triturada y su inclusión como agregado fino. Se evalúa el comportamiento de durabilidad del material mediante ataques químicos como sulfatación, carbonatación y reacción álcaliagregado. Además, se analiza el coeficiente de absorción de sonido y el índice de reducción de ruido del concreto con diferentes porcentajes de sustitución de arena por EHC.

\section{METODOLOGÍA}

Se realizó la dosificación de diseño de mezcla por volumen, utilizando el método estandarizado por el Instituto Americano del Concreto (ACI 221) con base en una resistencia de diseño de $14 \mathrm{Mpa}$ (2000 psi), relación agua/cemento de 0,68 y 28 días de curado. Se fabricaron probetas cilíndricas para cada uno de los ensayos de durabilidad, con $10 \mathrm{~cm}$ de diámetro y $20 \mathrm{~cm}$ de longitud. Los porcentajes de sustitución de escoria por agregado fino fueron de $0,10,15$ y $20 \%$, bajo las condiciones de la norma NTC 1377. Para los ensayos acústicos se fabricaron muestras con forma de disco, de $10 \mathrm{~cm}$ de diámetro y $2,5 \mathrm{~cm}$ de espesor para los mismos porcentajes de sustitución.

\section{Caracterización física}

Tanto el agregado grueso como el agregado fino y la escoria EHC se caracterizaron a partir de los ensayos descritos en la NTC 92, 176 y 237, los cuales permitieron calcular el peso unitario, la gravedad específica, el porcentaje de absorción y el porcentaje de vacíos. La granulometría de la arena, la EHC y el módulo de finura se determinaron según la NTC 77.

\section{Caracterización química}

Se realizó el ensayo de Energía Dispersiva Espectroscópica de Rayos X (EDX) a tres muestras de EHC (escogidas al azar) antes de ser triturada. Se realizó, además, el ensayo de Difracción de Rayos X (DRX) con el objetivo de descartar reacciones nocivas y confirmar los resultados del ensayo de EDX. Se tomaron muestras de concreto con sustitución del $20 \%$ de escoria por arena, concreto sin sustitución y exclusivamente EHC. Las muestras fueron pulverizadas en un mortero de ágata y reducidas a un tamaño de $38 \mu \mathrm{m}$ (400 mesh). El espécimen seleccionado de cada muestra fue montado en un porta de aluminio mediante 
la técnica de llenado frontal. La toma de datos se realizó en un difractómetro de polvo, marca Bruker, modelo Advance con geometría Da-Vinci bajo las condiciones descritas en la tabla 1 .

Tabla 1. Condiciones para la caracterización química de las probetas

\begin{tabular}{|l|l|l|}
\hline Voltaje & 40 & {$[\mathrm{kV}]$} \\
\hline Corriente & 30 & {$[\mathrm{~mA}]$} \\
\hline Rendija de divergencia & 0,6 & {$[\mathrm{~mm}]$} \\
\hline Rendija soller primario & 2,5 & {$\left[^{\circ}\right]$} \\
\hline Muestreo & $0,01526-2 \theta$ & {$\left[^{\circ}\right]$} \\
\hline Rango de medición & $3,5-70-2 \theta$ & {$\left[^{\circ}\right]$} \\
\hline Radiación & CuKa1 & \\
\hline $\begin{array}{l}\text { Filtro } \\
\text { Detector }\end{array}$ & $\begin{array}{l}\text { Níquel } \\
\text { LynxEye }\end{array}$ & \\
\hline Tipo de barrido & A pasos & \\
\hline Tiempo de muestreo & 0,4 & Seg \\
\hline
\end{tabular}

Fuente: elaboración propia.

El análisis cualitativo de las fases presentes en la muestra se realizó mediante comparación del perfil observado con los perfiles de difracción reportados en la base de datos del International Centre for Diffraction Data (ICDD). El análisis cuantitativo de las fases encontradas para la muestra de concreto con sustitución de arena por escoria se realizó mediante el refinamiento del perfil por el método Rietveld.

\section{Ensayo de sulfatación}

Para este ensayo se preparó una solución acuosa de sulfato de sodio anhidro $\left(\mathrm{Na}_{2} \mathrm{SO}_{4}\right)$ con una concentración de $80 \mathrm{~g} / 1$, equivalente al 7,77\% de concentración, teniendo en cuenta la densidad del sulfato $\left(2,68 \mathrm{~g} / \mathrm{cm}^{3}\right)$. Este procedimiento se realizó 48 horas antes de la inmersión de las probetas. Se utilizó esta concentración con el fin de acelerar el ataque de sulfatación del concreto en condiciones más críticas que las de servicio. Las probetas se sumergieron en dos recipientes, cada uno con $1000 \mathrm{~g}$ de $\mathrm{Na}_{2} \mathrm{SO}_{4}$ disueltos en 12,5 litros de agua. Se hizo la lectura del peso de las probetas pasados 28 días de curado y 30 días de secado. Se efectuaron siete ciclos de 48 horas, cada ciclo compuesto por las etapas de inmersión, secado y enfriamiento. La inmersión se realizó durante 24 horas para cada ciclo. Se secaron las probetas al horno con un tiempo de 8 horas por ciclo a $105^{\circ}$ $\mathrm{C}$, con posterior enfriamiento al ambiente por 16 horas. Durante el ensayo se midió la variación de la masa de las probetas, tanto seca (al horno) como húmeda (s.s.s.).

\section{Ensayo de la reacción álcali-agregado}

Se realizó la lectura del peso de las probetas pasados los 28 días de curado y los 120 días de secado, empleando una balanza digital. Se sumergieron las probetas en agua a $80^{\circ} \mathrm{C}$ durante 12 horas y posteriormente se trasladaron a una solución de soda cáustica $(\mathrm{NaOH})$ con concentración ml normal, equivalente a $40 \mathrm{~g}$ por cada $900 \mathrm{ml}$ de agua a $80^{\circ} \mathrm{C}$, preparada con anticipación de 48 horas. Se utilizó un recipiente con diferencias de nivel en su base, que garantizó la difusión de la soda cáustica en la parte inferior de las probetas. La duración total de la inmersión fue de 16 días. Las probetas solo se extrajeron de la solución mientras se realizaba el control de variación de masa, el cual se efectuó con balanza digital, cada 2 días en estado saturado superficialmente seco. Pasados los 16 días se evaluó la resistencia a compresión de las probetas utilizando el equipo MTS 637 Hydraulic Wedge Grip. Para los dos ensayos descritos anteriormente se realizó, además, una inspección visual de los posibles cambios en el aspecto exterior de las probetas.

\section{Ensayo de carbonatación acelerada}

Se utilizó un equipo que incrementa artificialmente la velocidad de reacción por medio del 
aumento de la concentración de $\mathrm{CO}_{2}$ en una atmósfera controlada. Para su puesta en marcha, la cámara requirió la alimentación de $\mathrm{CO}_{2}$, aire seco y aire húmedo. La fuente de $\mathrm{CO}_{2}$ es un tanque comercial de $25 \mathrm{~kg}$ con medidor de presión. El aire que ingresa a la cámara tiene como fuente el aire atmosférico, el cual es capturado por un compresor para aumentar la presión y dirigir el flujo. El ensayo de exposición al $\mathrm{CO}_{2}$ consistió en someter probetas de concreto secas (al ambiente por 240 días), con diferentes porcentajes de sustitución de arena por escoria en la cámara de carbonatación. La tabla 2 muestra las condiciones de humedad, temperatura y concentración para que se presente carbonatación.

Tabla 2. Condiciones de la cámara para la carbonatación acelerada del concreto

\begin{tabular}{|l|l|}
\hline Humedad relativa [\%] & $50-70$ \\
\hline Temperatura [ $\left.{ }^{\circ} \mathrm{C}\right]$ & 25 \\
\hline Concentración [\%] & 70 \\
\hline Tiempo del ensayo [hrs] & 15 \\
\hline
\end{tabular}

Fuente: elaboración propia.

Se secó totalmente la cámara, se llenó de agua el recipiente que alimenta la caja de acero inoxidable para la correcta medición de humedad y se ingresaron las probetas. Se fijó el valor de la histéresis de humedad en $65 \%$. El controlador de tiempo se estableció en 15 horas, teniendo en cuenta la alta concentración. Posteriormente, se energizó la cámara y se abrió la válvula del tanque de $\mathrm{CO}_{2}$ a una presión de 250 psi, que garantizó el flujo constante. Se permitió solo el paso de $\mathrm{CO}_{2}$, sin entrada de aire a la cámara, hasta alcanzar el valor de histéresis; luego se abrió la válvula de aire seco para su estabilización. Se fijó el flujo de aire húmedo inicial en cero, debido a la humedad relativa del ambiente, la cual varió entre 80 y $85 \%$. Al descender el valor de la humedad relativa hasta menos del $75 \%$, se abrió la válvula de aire húmedo. Se garantizó la concentración del $70 \%$ periódicamente con el medidor de concentración de $\mathrm{CO}_{2}$. Al finalizar las 15 horas de carbonatación, se cerró el paso de $\mathrm{CO}_{2}$ y se dejó la cámara en operación por una hora más, solo con flujo de aire seco para poder extraer las probetas de forma segura. Se realizó un ensayo de tracción indirecta a las probetas sometidas a carbonatación acelerada, según la NTC 722. Aprovechando la forma de la falla, se aplicó fenolftaleína al 1\% en solución etílica para determinar las zonas con $\mathrm{pH}$ básico (las no carbonatadas presentan color fucsia) y las zonas con $\mathrm{pH}$ ácido (las carbonatadas mantienen el color del concreto), ya que el $\mathrm{CO}_{2}$ es un compuesto ácido y el concreto es un compuesto básico. Se realizaron las mediciones en cada probeta, registrando los valores máximos y mínimos de profundidad de carbonatación $X$ para establecer el coeficiente de carbonatación $K$ y así estimar una edad de vida útil de cada probeta, el cual se asumió como el tiempo que tarda el $\mathrm{CO}_{2}$ en difundirse dentro del concreto, hasta llegar al acero de refuerzo. Se tomó una profundidad $X$ de $40 \mathrm{~mm}$, ya que el refuerzo mínimo para vigas y columnas en armadura principal, estribos y espirales es de $40 \mathrm{~mm}$, según la norma NSR-10 (C.7.7.1).

\section{Evaluación de las propiedades acústicas}

El índice de absorción del sonido (NRC) es el valor que indica qué tanto sonido absorbe determinado material y puede determinarse a partir de la ecuación (1)

$$
N C R=\frac{\sum \alpha}{n}
$$

El coeficiente de absorción del sonido $(\alpha)$ del concreto modificado se determinó por medio del ensayo del tubo de impedancia (ver figura 1). El equipo consiste en un tubo cerrado de PVC con sección transversal circular de $10 \mathrm{~cm}$ de diámetro, provisto de un parlante ubicado en el extremo opuesto a la 


\section{investigación}

muestra y un sistema interno de micrófono móvil unidireccional de $18 \mathrm{~mm}$ de diámetro.

El ensayo tiene como fundamento físico la teoría de ondas estacionarias en un tubo cerrado. El límite superior de frecuencias que es preciso utilizar en el ensayo y el diámetro del tubo están relacionados por la desigualdad de la ecuación (2), según la norma ASTM C 384.

$$
f<0,586=\frac{v s}{d}
$$

En la ecuación (2) $f$ es la frecuencia máxima permitida en el ensayo medida en Hertz, $v s$ es la velocidad del sonido $(343 \mathrm{~m} / \mathrm{s})$ y $d$ es el diámetro del tubo $(0,107 \mathrm{~m})$. Las frecuencias utilizadas en el ensayo fueron: 250, 315, 400, 630, 800, 1000, 1250 y $1600 \mathrm{~Hz}$, todas menores de 1878,5 Hz.

El ensayo se realizó con los parámetros de la ASTM C 384, utilizando el tubo de impedancia, un osciloscopio marca Tektronic de referencia TDS 200 2B y un generador de señales marca Protek de referencia $9205^{\mathrm{a}}$ (ver figura $2 \mathrm{a}$ y $2 \mathrm{~b}$ ). Las muestras de concreto con EHC se ponen en un extremo del tubo de impedancia, el parlante con su soporte en el otro extremo y el micrófono desplazándose a lo largo del tubo por medio del sistema de varilla móvil. Enseguida se generan las señales y se miden las magnitudes de la presión en los nodos y antinodos con el micrófono de prueba.
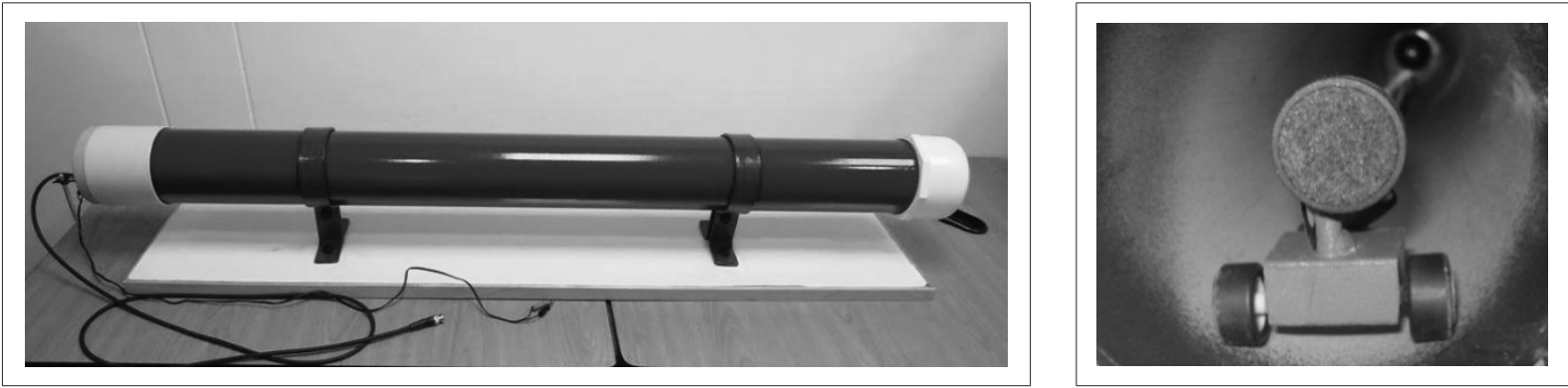

Figura 1 (a-b). Tubo de impedancia

Fuente: elaboración propia.
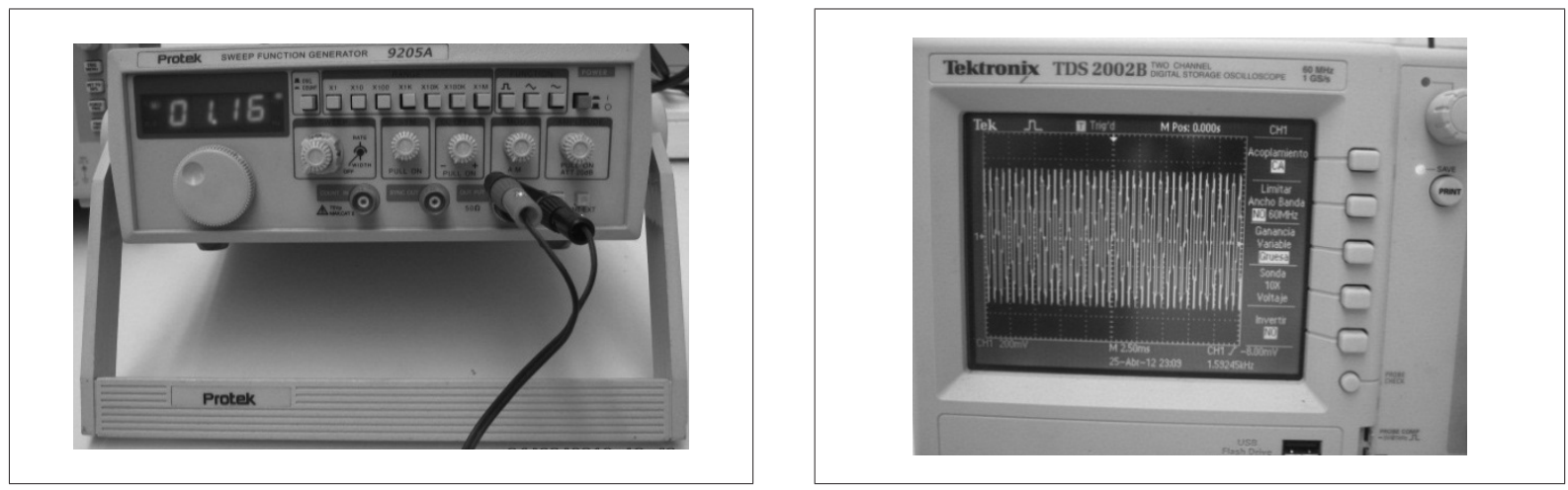

Figura 2 (a-b). Generador de señales y osciloscopio en el desarrollo del ensayo

Fuente: elaboración propia. 


\section{investigación}

\section{RESULTADOS}

\section{Caracterización física}

La tabla 3 muestra los resultados obtenidos del peso unitario, el porcentaje de vacíos (NTC 92), la gravedad específica y el porcentaje de absorción del agregado grueso (NTC 176), arena y escoria (NTC 237). El porcentaje de humedad se calculó según la INV E - 122. Se determinó además, la curva granulométrica del agregado fino, tanto para la arena (figura 3a) como para la EHC (figura 3b) y el módulo de finura mediante la NTC 77.

El sistema de clasificación unificado de suelos (USCS) indicó que la arena utilizada es de tipo SP. Su módulo de finura fue de 2,073 y se encuentra en el rango de "arenas finas". Según el USCS, la escoria triturada utilizada es de tipo SW. Su módulo de finura fue de 3,413 , por lo que se concluye que se trata de una "arena gruesa".

Tabla 3. Gravedades específicas y pesos unitarios de los agregados

\begin{tabular}{|l|c|c|c|}
\hline \multicolumn{1}{|c|}{ Gravedades específicas y pesos unitarios } & Ag. grueso & Ag. fino & EHC triturada \\
\hline Gravedad específica real $[\mathrm{g} / \mathrm{g}]$ & 2,72 & 2,71 & 2,40 \\
\hline Gravedad específica aparente [g/g] & 2,61 & 2,58 & 2,35 \\
\hline Gravedad específica aparente S.S.S [g/g] & 2,65 & 2,63 & 2,37 \\
\hline Peso unitario del agregado S.S.S. compactado $\left[\mathrm{kg} / \mathrm{m}^{3}\right]$ & 1477,79 & 1652,33 & 1727,43 \\
\hline Peso unitario del agregado S.S.S. suelto $\left[\mathrm{kg} / \mathrm{m}^{3}\right]$ & 1345,56 & 1392,98 & 1437,94 \\
\hline Absorción [\%] & 1,56 & 1,87 & 0,85 \\
\hline Vacíos [\%] & 44,29 & 37,21 & 27,10 \\
\hline Humedad [\%] & 0,49 & 0,83 & 0,54 \\
\hline
\end{tabular}

Fuente: elaboración propia.

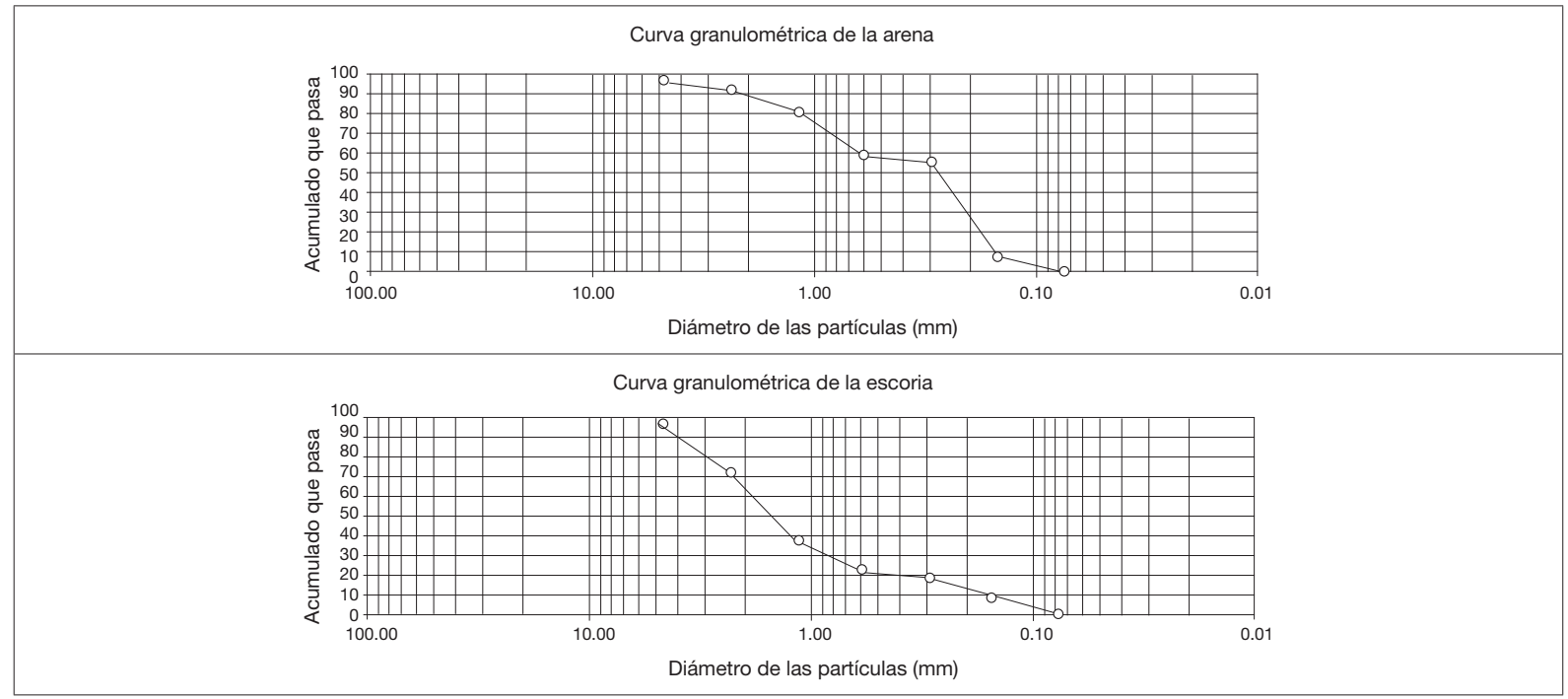

Figura 3. a) Curva granulométrica de la arena b) Curva granulométrica de la escoria de horno de cubilote triturada Fuente: elaboración propia. 


\section{Caracterización química}

En la figura 4 se observa que según el método de análisis de energía dispersiva espectroscópica de rayos X (EDX), los elementos que principalmente componen la EHC son silicio y calcio.

Las fases de la EHC no modifican cualitativamente la conformación de fases principales del concreto con reemplazo del $20 \%$, ya que la mayoría no son cuantificables debido a su baja proporción. Sin embargo, el porcentaje de sílice en forma de agregado aumenta, por lo que resulta determinante. Este planteamiento se verifica por medio del análisis cuantitativo (tabla 4).

Se comprobó la influencia de los elementos principales de la EHC en las fases cristalinas del material compuesto. Se determinó un alto contenido de sílice en forma de agregado, lo cual, potencialmente puede causar un efecto de arenas expansivas al contacto con el agua (ver figura 5).

\section{Ensayo de sulfatación}

En la figura 6 se observa que los últimos ciclos de humedecimiento y secado causaron un desprendimiento de material y el surgimiento de una capa de sulfato solidificado alrededor de las probetas, generado por el ascenso del sulfato hacia la superficie debido a acciones capilares y a la cristalización de sales por evaporación. Esta acción resultó ser la causa principal del daño presentado en las probetas (ver figura 7).

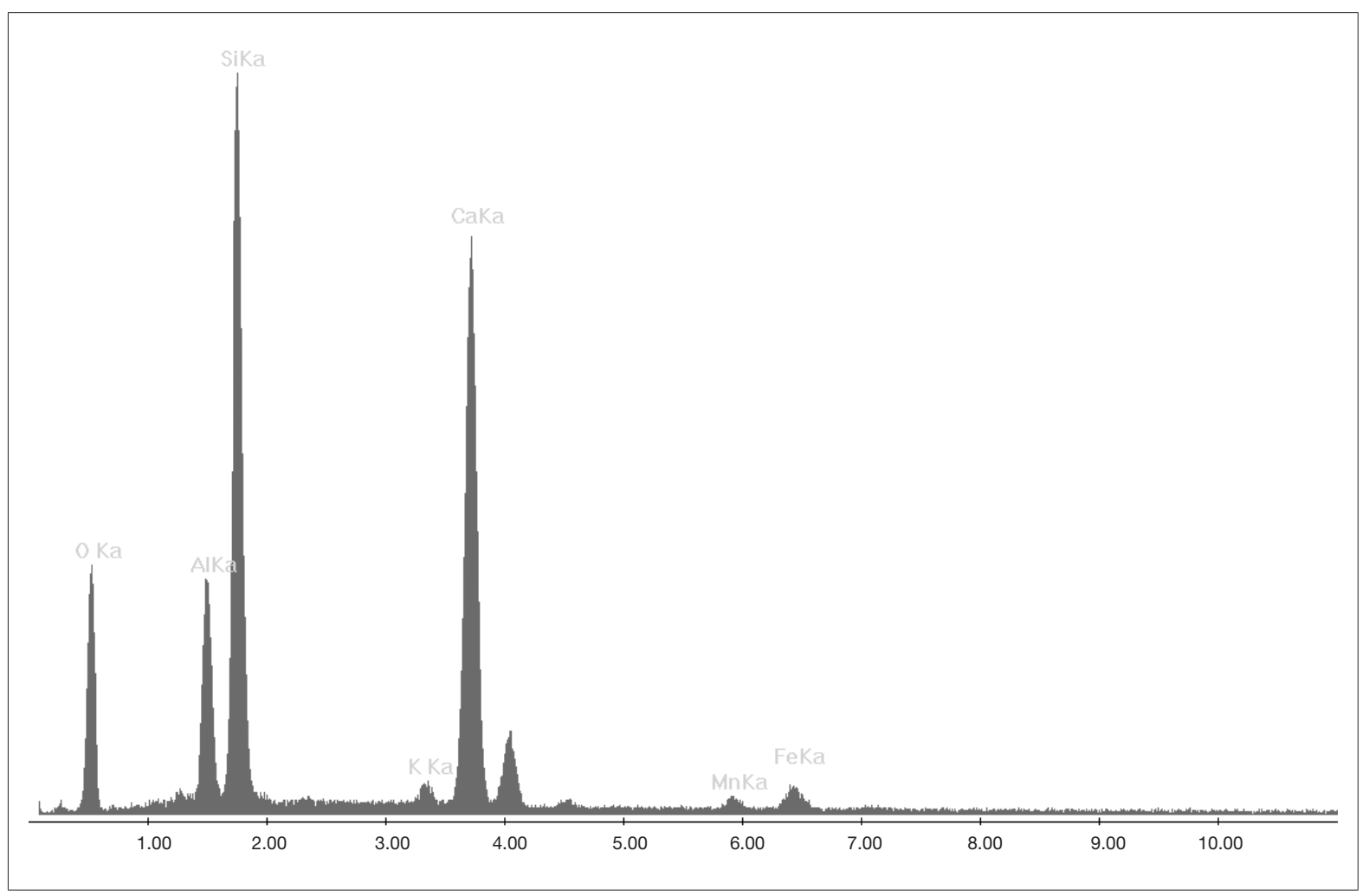

Figura 4. Espectro de rayos $x$ de la EHC

Fuente: elaboración propia. 


\section{investigación}

Tabla 4. Análisis DRX cuantitativo del concreto con sustitución de agregado fino por escoria al $20 \%$

\begin{tabular}{|c|c|c|c|}
\hline \multicolumn{2}{|r|}{ Fase } & \multirow[t]{2}{*}{ Nombre } & \multirow{2}{*}{\begin{tabular}{|l|} 
Porcentaje cuantitativo (D. E.) \\
$22,1(0,2)$ \\
\end{tabular}} \\
\hline \multirow{13}{*}{ Cristalinos } & $\mathrm{SiO}_{2}$ & & \\
\hline & $\mathrm{CaCO}_{3}$ & Calcita & $35,8(0,3)$ \\
\hline & $\mathrm{Ca}(\mathrm{OH}) 2$ & Portlandita & $2.0(0,0)$ \\
\hline & $\mathrm{Ca}\left(\mathrm{CO}_{3}\right)$ & Vaterita & $5,3(0,1)$ \\
\hline & $\mathrm{Al} 2\left(\mathrm{Si}_{2} \mathrm{O}_{5}\right)(\mathrm{OH})_{4}$ & Caolinita & $2,6(0,1)$ \\
\hline & $(\mathrm{Fe}, \mathrm{Al}) \mathrm{PO}_{4} \cdot{ }_{3} \mathrm{H}_{2} \mathrm{O}$ & Koninckita & N.C \\
\hline & $\mathrm{Fe}_{3} \mathrm{Si}$ & Gupeita & N.C \\
\hline & $\mathrm{Ca}_{3} \mathrm{SiO} 5$ & Hatrurita & $5,0(0,2)$ \\
\hline & $\mathrm{MnS}$ & Sulfuro de Manganeso & N.C \\
\hline & $\mathrm{KAl} \mathrm{Si}_{3} \mathrm{O}_{8}$ & Microclina & N.C \\
\hline & $\mathrm{KAl}_{2}\left(\mathrm{Si}_{3} \mathrm{Al}\right) \mathrm{O}_{10}(\mathrm{OH})_{2}$ & Moscovita & $13,8(0,3)$ \\
\hline & $\mathrm{Na}\left(\mathrm{Al} \mathrm{Si}{ }_{3} \mathrm{O}_{8}\right)$ & Albita & $4,6(0,2)$ \\
\hline & $(\mathrm{Mg}, \mathrm{Fe})_{6}(\mathrm{Si}, \mathrm{Al})_{4} \mathrm{O} 10(\mathrm{OH}) 8$ & Clinocloro & $2,4(0,1)$ \\
\hline \multicolumn{3}{|c|}{ Total Cristalino } & 93,6 \\
\hline \multicolumn{3}{|c|}{ Amorfos y otros } & 6.4 \\
\hline
\end{tabular}

Nota: N.C = no cuantificable (debido a su baja proporción). D.E. = desviación estándar.

Fuente: elaboración propia.

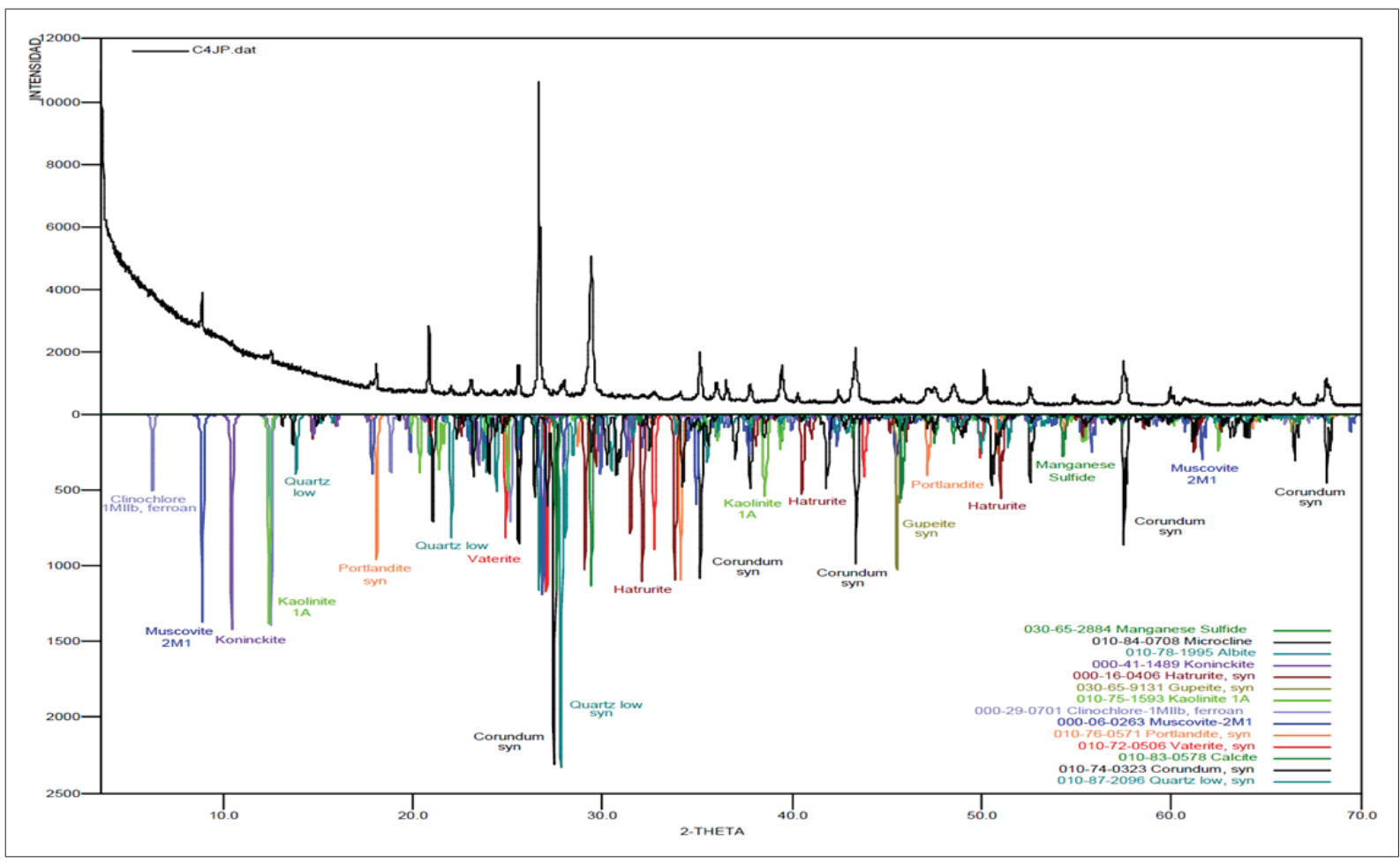

Figura 5. Espectro de rayos $\mathrm{X}$ del material compuesto

Fuente: elaboración propia. 


\section{investigación}

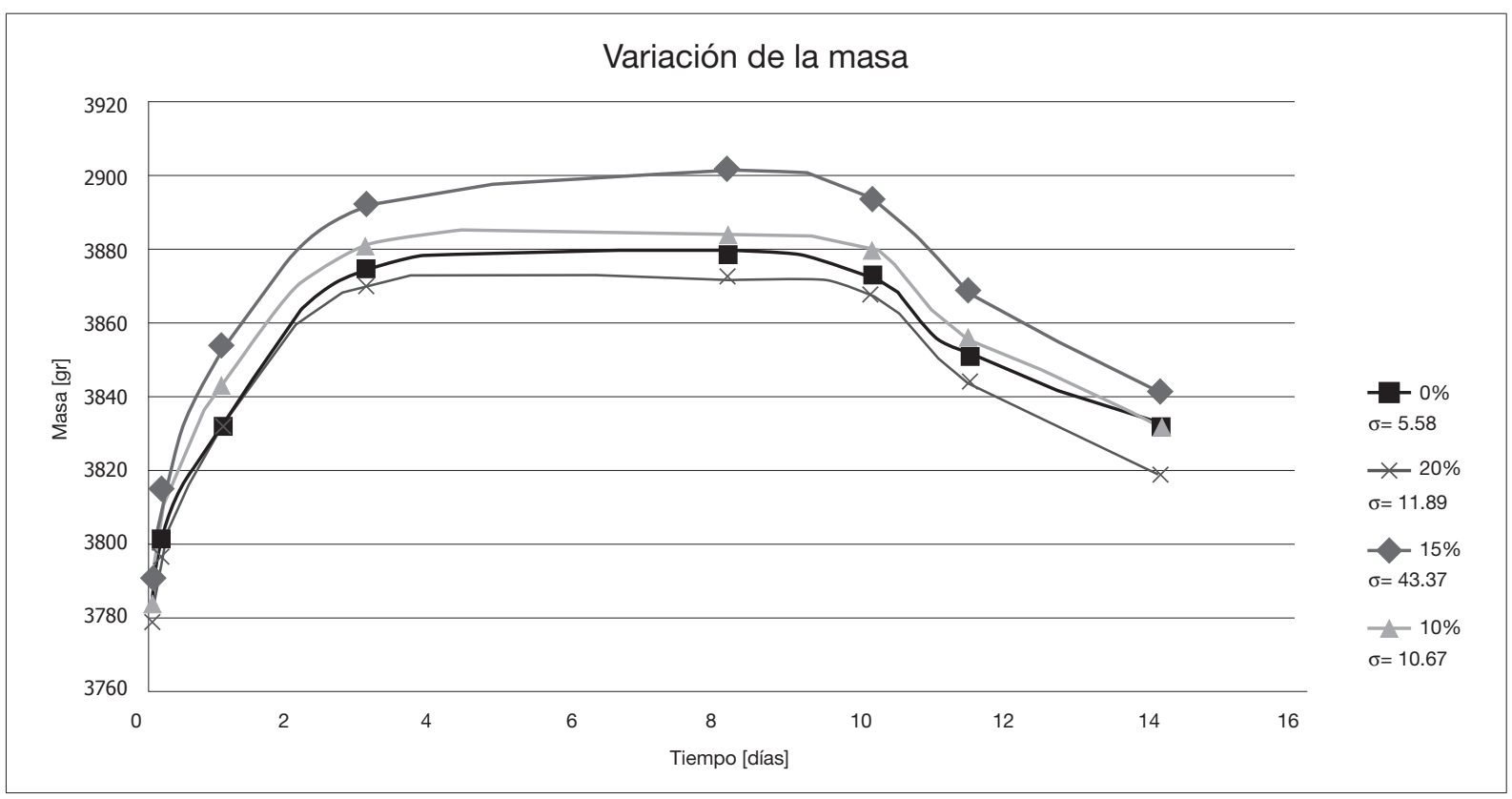

Figura 6. Variación de la masa húmeda de las probetas sometidas a sulfatación

Fuente: elaboración propia.
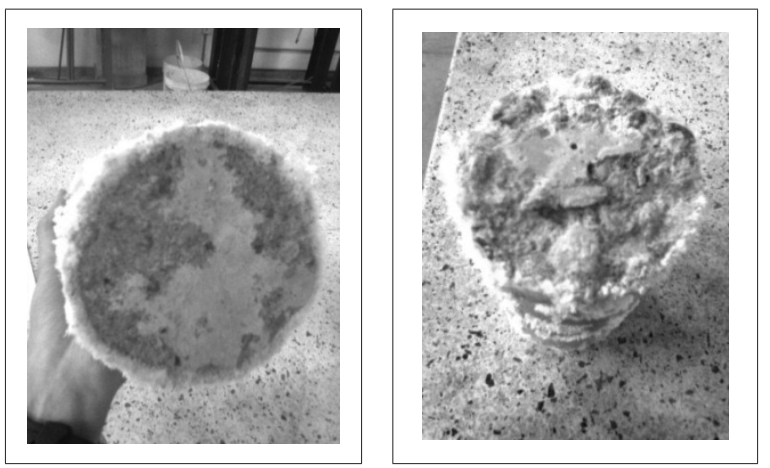

Figura 7. a) Cristalización de sales b) Daño generado en una probeta con sustitución del $20 \%$

Fuente: elaboración propia.

\section{Ensayo de la reacción álcali-agregado}

En este ensayo no se establecieron etapas de secado, no se presentó una variación considerable por saturación de poros y se determinó que las probetas con el $20 \%$ de sustitución de arena por EHC tuvieron el mayor porcentaje de aumento de masa, como lo muestra la figura 8 .

La variación de la masa indica que hay mayor difusión de la solución de soda cáustica en el concreto a mayor reemplazo de escoria por arena. Este fenómeno se relaciona con la reacción entre los álcalis del cemento y los agregados con alto contenido de sílice. La reacción induce una expansión de la estructura del concreto con posibles agrietamientos internos. Las probetas mostraron cambios en la textura de la superficie, volviéndose resbaladiza.

Los resultados del ensayo de resistencia a la compresión muestran que las probetas con porcentaje de sustitución del $20 \%$ presentaron mayor disminución de la resistencia que las probetas con menor porcentaje de sustitución (ver figura 9). 


\section{investigación}

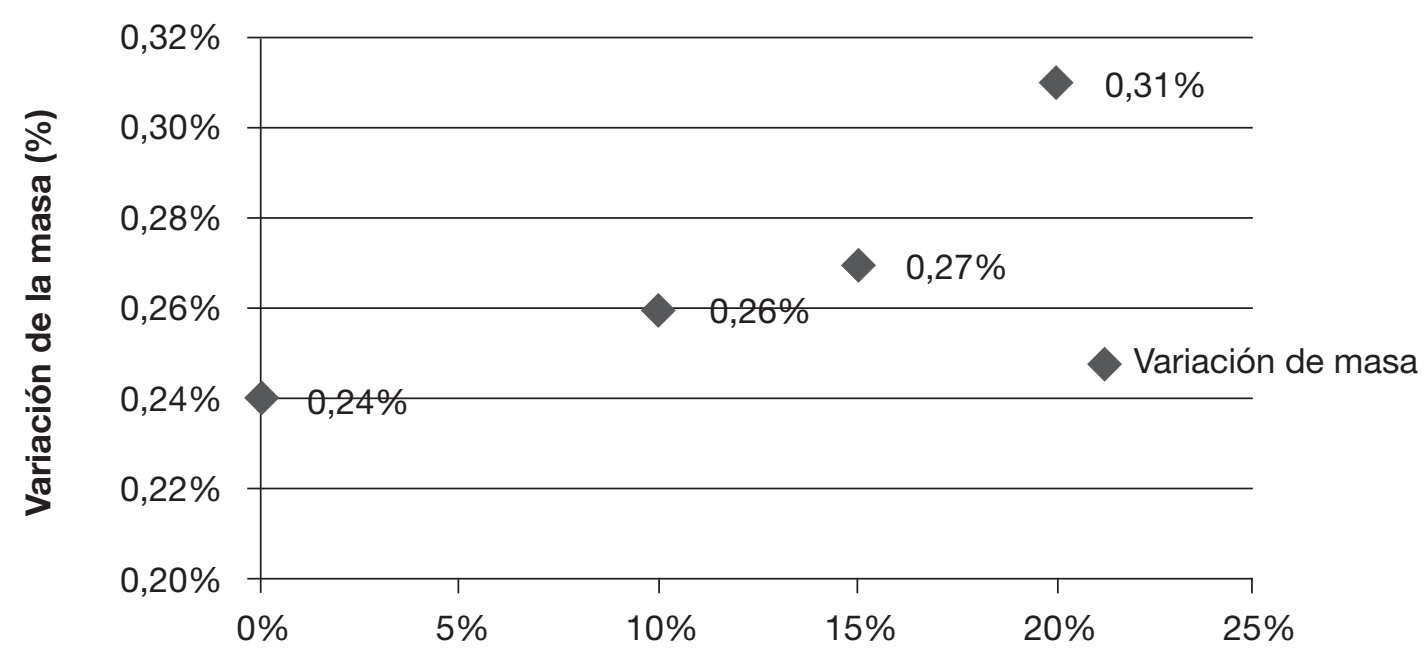

(\%) Sustitución EHC por arena

Figura 8. Porcentaje de variación de la masa respecto al porcentaje de sustitución Fuente: elaboración propia.

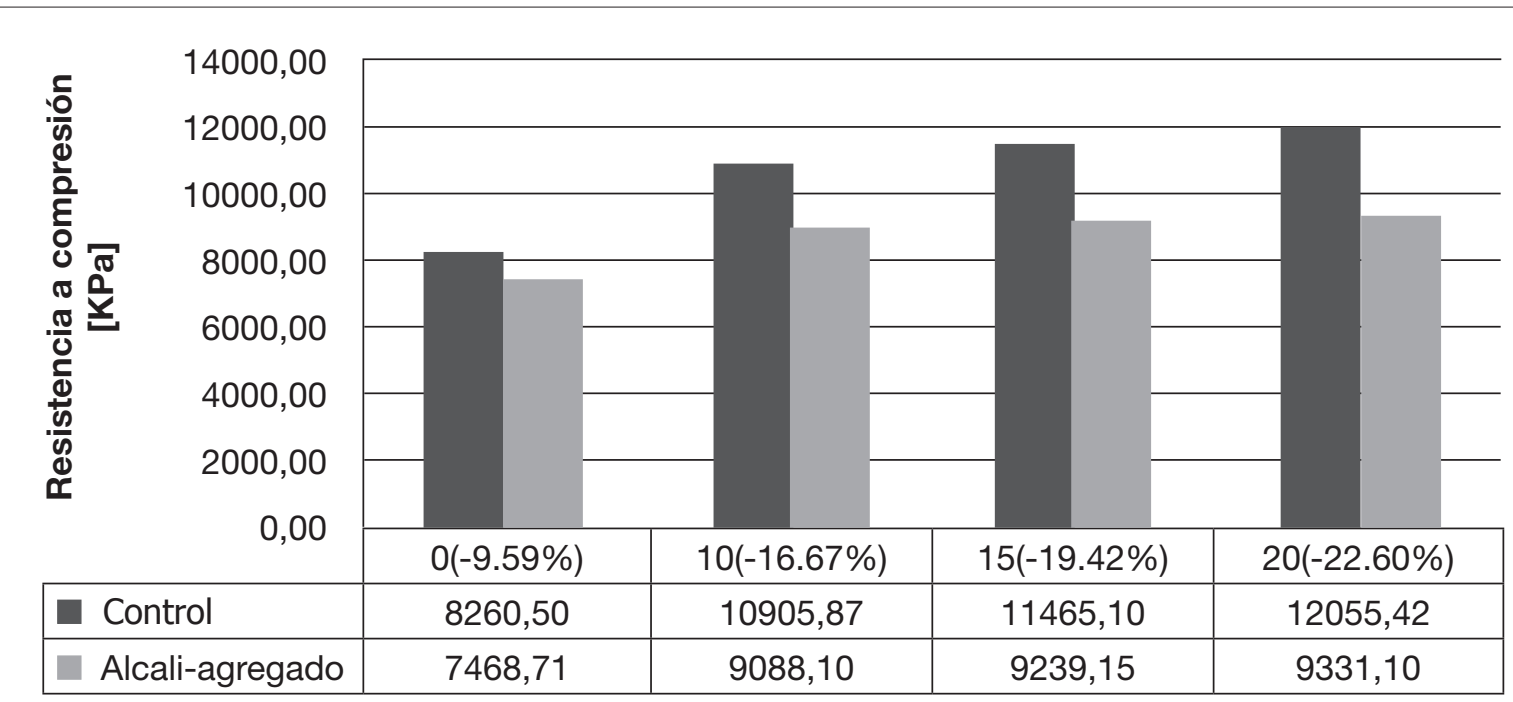

Figura 9. Resultados del ensayo de resistencia a la compresión posterior al de RAA

Fuente: elaboración propia. 


\section{Ensayo de carbonatación acelerada}

En la figura 10 se observan los resultados obtenidos de la medición de la profundidad de penetración por medio del análisis de $\mathrm{pH}$.

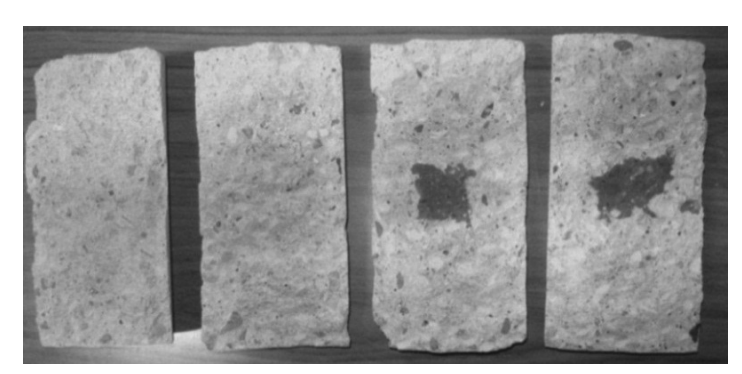

Figura 10. Profundidades de carbonatación para 0 , 10,15 y $20 \%$ de sustitución

Fuente: elaboración propia.

A la zona donde no se presenta color fucsia se le llama zona carbonatada, puesto que esta solución es incolora en $\mathrm{pH}$ inferiores a 8,5. Para valores de $\mathrm{pH}$ superiores a 9,5 se torna de color púrpura y entre 8,5 y 9,5 el indicador adquiere una coloración de color rosa al rojo-púrpura (Moreno, Domínguez, Cob y Duarte, 2004). En cuanto al $\mathrm{pH}$, en las probetas con el $0 \%$ de sustitución se evidenció una difusión completa del $\mathrm{CO}_{2}$, fueron carbonatadas completamente con $\mathrm{pH}$ menor de 8,5 ; las probetas con sustitución del $10 \%$ se carbonataron completamente con un $\mathrm{pH}$ entre el 8,5 y el 9,5, y las probetas con sustitución del 15 y $20 \%$ fueron carbonatadas pero no completamente, estas tuvieron zonas no carbonatadas con un $\mathrm{pH}$ mayor de 9,5.

El coeficiente de carbonatación se determinó a partir de $X=K \sqrt{t}$, utilizando $\mathrm{x}=40 \mathrm{~mm}$, que es el recubrimiento mínimo exigido en la NSR (10) para vigas y columnas (figura 11 y 12). El tiempo de vida útil estimado para una estructura de concreto de EHC con diferentes porcentajes de sustitución se calculó mediante la expresión $T_{1}=T_{2} \frac{C S_{2}}{C s_{1}}$ (ver figura 13). Se realizó una transformación del tiempo de vida útil con concentración del 70\% a un tiempo de vida útil, a una concentración del medio ambiente $0,03918 \%$ de concentración $\mathrm{CO}_{2}$ $\left(\mathrm{CO}_{2}, 2011\right)$.

Ensayo acústico por el método del tubo de impedancia: se determinó el coeficiente de absorción acústica $\left(\alpha=1-|r|^{2}\right)$ y el índice de reducción de ruido (NRC) del concreto modificado con EHC. El coeficiente de absorción para los rangos de frecuencias entre 400 y $1200 \mathrm{~Hz}$ de los concretos modificados con EHC a los diferentes porcentajes, es más alto que el del concreto normal, siendo el mejor valor el de la sustitución al 20\%. Esto indica que el concreto modificado es mejor absorbente del sonido para este rango de frecuencias, como se observa en la figura 14. Igualmente, la capacidad de material de absorción del sonido se puede expresar a través del coeficiente de reducción de ruido NCR. Los resultados muestran claramente que el concreto modificado, en particular aquel al $20 \%$, tiene mejores resistencias al sonido $(12 \%$ mejor) que el concreto sin modificar, como se observa en la figura 15.

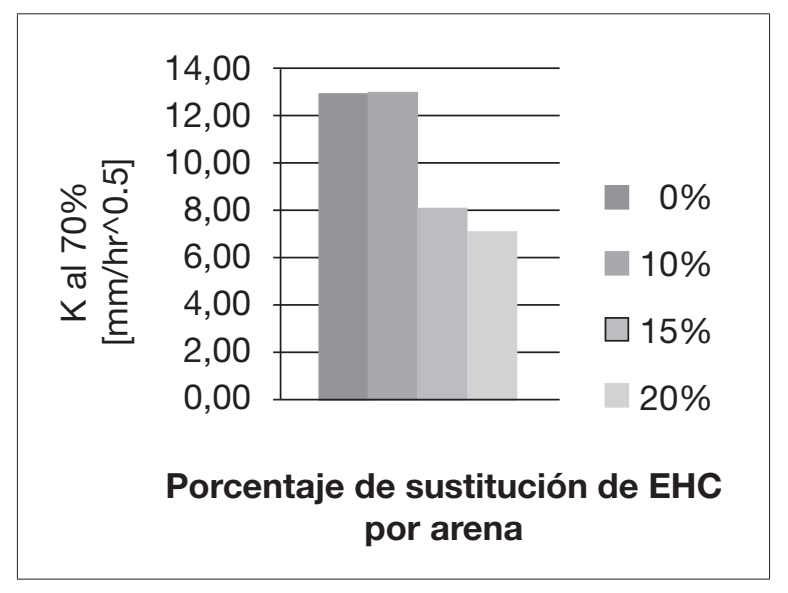

Figura 11. Coeficiente de carbonatación $[\mathrm{K}]$ para una concentración del $70 \%$

Fuente: elaboración propia. 


\section{investigación}

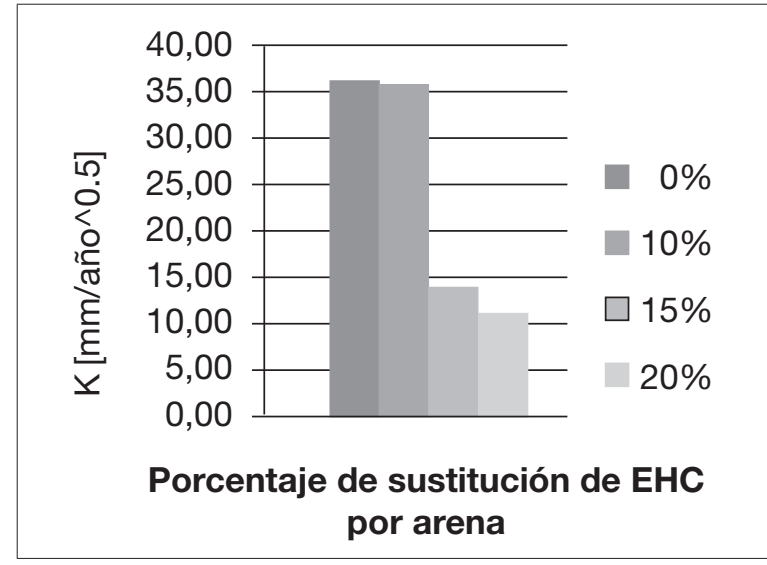

Figura 12. Coeficiente de carbonatación $[\mathrm{K}]$ a concentración ambiente [0,039\%]

Fuente: elaboración propia.

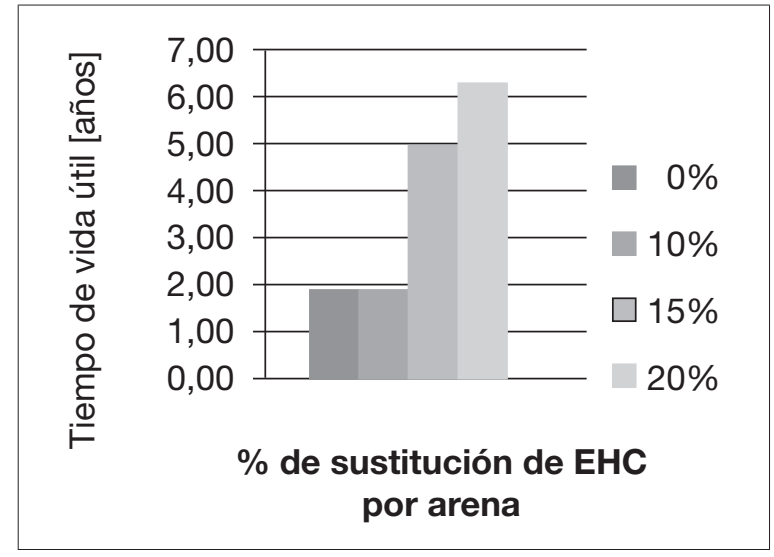

Figura 13. Tiempo de vida útil estimado para una estructura de concreto de EHC

Fuente: elaboración propia.

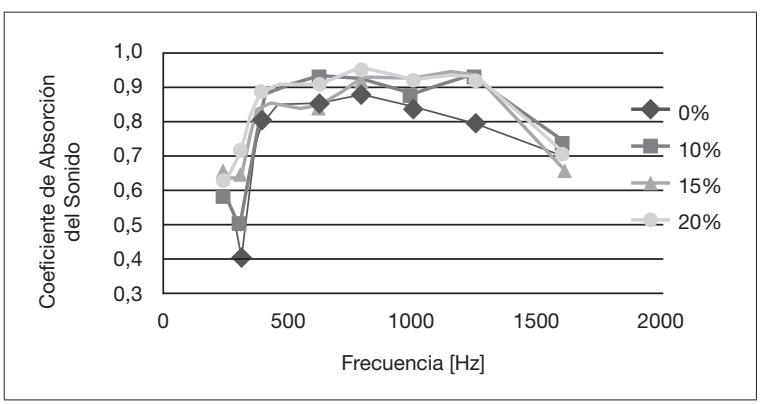

Figura 14. Coeficiente de absorción acústico medido a distintas frecuencias

Fuente: elaboración propia.

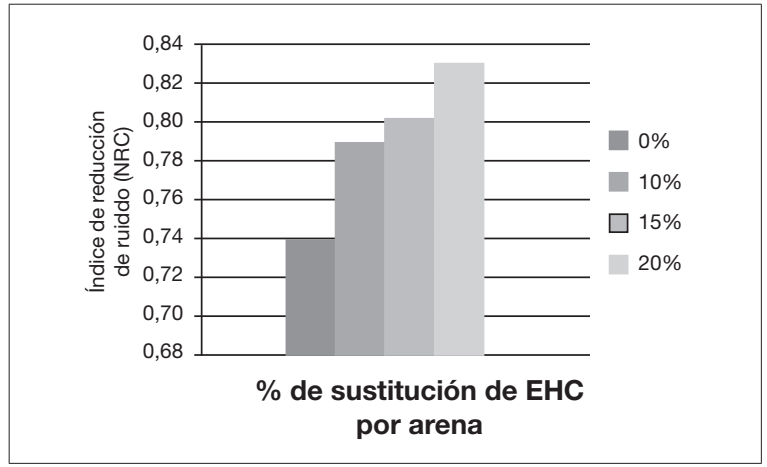

Figura 15. Índice de reducción de ruido (NRC) para diferentes sustituciones de escoria por arena

Fuente: elaboración propia.

\section{CONCLUSIONES}

La clasificación granulométrica realizada permitió caracterizar a la EHC como arena gruesa bien gradada con pocos finos. Se obtuvieron ciertas diferencias en sus propiedades físicas y granulometría respecto de los agregados.

Según el ensayo de EDX, los elementos que principalmente componen la EHC son sílice y calcio, los cuales también están presentes en el concreto convencional en diferentes compuestos, por lo que a priori son materiales químicamente compatibles.

El ensayo de DRX comprueba que las fases de la EHC no influyen cualitativamente en la conformación de fases principales del material compuesto; sin embargo, contribuyen al aumento de sílice en forma de agregado, lo que puede producir un efecto de arenas expansivas en ambientes húmedos.

No es recomendable la utilización del concreto de EHC como agregado fino para elementos sometidos a medios húmedos con sulfatos, sobre todo si se presentan ciclos de humedecimiento y secado, pues se determinó que con porcentaje de 
sustitución del $20 \%$ ocurrió una pérdida de masa de $2,47 \%$, contra un $1,07 \%$ de pérdida sin reemplazo. El desprendimiento de la pasta fue proporcional al porcentaje de sustitución, y fue causado por la cristalización de sales en la superficie de las probetas.

Existe influencia de la reacción entre los álcalis del cemento y el agregado fino de EHC en la difusión de la solución de soda cáustica en concretos con mayor porcentaje de EHC $(0,31 \%$ de aumento de masa para el $20 \%$, versus $0,24 \%$ sin $\mathrm{EHC})$, por su alto contenido de sílice. La reacción induce una expansión de la estructura del concreto, con posibles agrietamientos internos. No se recomienda utilizar EHC como agregado fino en medios acuosos donde haya presencia inminente de la reacción álcali-agregado.

El agregado fino de EHC favorece la disminución de la difusión de $\mathrm{CO}_{2}$ en el concreto. Las probetas con $20 \%$ de sustitución indicaron que la edad de vida útil de estructuras de concreto reforzado con este porcentaje de EHC es aproximadamente 10 veces mayor que la de estructuras de concreto sin escoria en ambientes carbonatados.

La escoria de horno de cubilote como agregado fino contribuye a la absorción acústica del concreto. A mayor porcentaje de sustitución de escoria por arena, mayor es el índice de reducción de ruido (NRC). Se presentó un índice de 0,83 para el concreto modificado al $20 \%$ de sustitución en comparación con el NRC de 0,74 correspondiente al concreto sin modificar, lo que podría ser ventajoso al fabricar elementos para el aislamiento acústico con concretos base EHC.

\section{FINANCIAMIENTO}

Vicerrectoría de Investigación y Extensión de la Universidad Industrial de Santander, proyecto 5561 "Uso de la escoria de procesos metalúrgicos como agregado del concreto para la fabricación de elementos de construcción no estructurales- Fase I".

\section{REFERENCIAS}

Anastasiou, E., Georgiadis, K. y Stefanidou, M. (2014). Utilization of Fine Recycled Aggregates in Concrete with Fly Ash and Steel Slag. Construction and Builing Materials, 50, 154-161.

Baricová, D., Pribulová, A. y Demeter, P. (2010). Comparison of Possibilites the Blast Furnance and Cupola Slag Utilization by Concrete Production. Archives of Foundary Engineering, 10 (1), 15-18.

Blissett, R. S. y Rowson, N. A. (2012). A Review of the Multi-Component Utilisation of Coal Fly Ash. Fuel, 97, 1-23.

$\mathrm{CO}_{2}$ Now (2011). Earth's $\mathrm{CO}_{2}$. Recuperado de http://co2now.org/
Doutres, O., Salissou, Y., Atalla, N. y Panneton, R. (2010). Evaluation of the Acoustic and NonAcoustic Properties of Sound Absorbing Materials Using a Three-Microphone Impedance Tube. Applied Acoustics, 71 (6), 506-509.

Kim, H. K. y Lee, H. K. (2010). Acoustic Absorption Modeling of Porous Concrete Considering the Gradation and Shape of Aggregates and Void Ratio. Journal of Sound and Vibration, 329 (7), 866-879.

Kim, H. K. y Lee, H. K. (2010). Influence of Cement Flow and Aggregate Type on the Mechanical and Acoustic Characteristics of Porous Concrete. Applied Acoustics, 71, 607-615. 


\section{investigación}

Leiva, C., Solís-Guzman, J., Marrero, M. y García Arena, C. (2013). Recycled Blocks with Improved Sound and Fire Insulation Containing Construction and Demolition Waste. Waste Management, 33 (3), 663671.

Mohammed, N. y Pofale, A. D. (2012). Experimental Investigation of Using Slag as an Alternative to Normal Aggregates (Coarse and Fine) in Concrete. International Journal of Civil ans Structural Engineering, 3 (1), 117-127.

Moreno, E., Domínguez, G., Cob, E. y Duarte, F. (2004). Efecto de la relación agua/cemento en la velocidad de carbonatación del concreto utilizando una cámara de aceleración. Ingeniería, 8 (2), 117-130.

Naik, T. (2002). Greener Concrete Using Recycled Materials. Concrete International, 24 (7), 45-49.

Park, S. B., Seo, D. S. y Jun, L. (2005). Studies on the Sound Absorption Characteristics of Porous Concrete Based on the Content of Recycled Aggregate and Target Void Ra- tio. Cement and Concrete Research, 35 (9), 1846-1854.

Pastor, J. M., García, L. D., Quintana, S. y Peña, J. (2014). Glass Reinforced Concrete Panels Containing Recycled Tyres: Evaluation of the Acoustic Properties of for their Use as Sound Barriers. Construction and Building Materials, 54, 541-549.

Ramamurthy, K., Kunhanandan, E. K. y Indu Siva, G. (2009). A Classification of Studies on Properties of Foam Concrete. Cement and Concrete Composites, 31, 388-396.

Tiwari, V., Shukla, A. y Bose, A. (2004). Acoustic Properties of Cenosphere Reinforced Cement and Asphalt Concrete. Applied Acoustics, 65, 263-275.

Zaetang, Y., Wongsa, A., Sata, V. y Chindaprasirt, P. (2013). Use of Lightweight Aggregates in Previous Concrete. Construction and Building Materials, 48, 585-591.

Zakaria, M. y Cabrera, J. G. (1996). Performance and Durability of Concrete Made with Demolition Waste and Artificial Fly Ash-Clay Aggregates. Waste Management, 16, 151-158. 